\title{
El Efecto de la Desigualdad Económica en los Homicidios en la Unión Europea*
}

\author{
Jonathan Torres-Tellez \\ ${ }^{1}$ Doctor en Ciencias Jurídicas y Políticas, Universidad Pablo de Olavide. Sevilla, España \\ E-mail: jonathantorrestellez@gmail.com
}

Base de datos: https://dataverse.harvard.edu/dataset.xhtml?persistentId=doi:10.7910/ DVN/PWNHRW

\section{INTRODUCCIÓN}

T a Unión Europea (UE) desde hace aproximadamente una década Lufre importantes problemas que están poniendo en duda el proyecto político y económico conocido hasta el momento y que puede producir en un futuro no muy lejano su fragmentación (Habermas, 2012; Pirro, Taggart y Van Kessel, , 2018).

Pero, sin embargo, existe una cuestión mucho más silenciosa que está socavando los pilares sociales sobre los que se ha construido la Unión Europea y que está mermando de manera considerable el apoyo ciudadano a este proyecto político: la desigualdad. Si bien no se trata de un fenómeno nuevo, ya que venía creciendo desde 1980, es cierto que la crisis financiera, a pesar de la evidente recuperación económica de los últimos años, la ha intensificado (OECD, 2017). No sólo han aumentado las diferencias entre los ricos y los más desfavorecidos en muchos países, sino que los países del sur, por ejemplo, han visto como sus indicadores económicos cada vez están más atrasados respecto a los del norte.

En este sentido, el informe "Finanzas de los Hogares" del Banco Central Europeo del 2016 afirmaba que el 10\% de los más ricos tenían en su poder el 51,2\% de la riqueza neta total de la Eurozona. Del mismo

"Agradecemos todas las sugerencias hechas por los evaluadores. 
modo, según datos de Eurostat, en 2018 las personas de la Unión Europea con mayor renta disponible recibían 5,2 veces más que el $20 \%$ que se encontraba en los deciles más bajos. Además, es importante recalcar que esta media ponderada de la totalidad de los países que conforman la UE enmascara importantes diferencias nacionales. Por ejemplo, en España esta brecha ha aumentado durante la crisis económica, pasando de recibir 5,5 veces más a 6,8; en Italia la diferencia pasó de 5,2 a 6,3; o en Lituania que en sólo 7 años aumentó esta distancia de 5,9 a 7,5.

Otra consecuencia importante de la crisis económica ha sido el aumento de la tasa de riesgo de pobreza en la UE que ha pasado del $14,7 \%$ en 2005 al 17,3\% en 2016 (Gráfico 1). La preocupación es mayor, si cabe, cuando este dato se desagrega a nivel nacional, ya que más de una quinta parte de la población de hasta 8 países está en riesgo de pobreza (Rumanía 25,3\%, Bulgaria 22,9\%, España 22,3\%, Lituania 21,9\%, Letonia $21,8 \%$, Estonia $21,7 \%$, Grecia $21,2 \%$ e Italia $20,6 \%$ ); o incluso Suecia, que ha destacado tradicionalmente por su bajo riesgo de pobreza, ha visto crecer esta variable de 10,1\% en 2007 a 15,8\% en 2018.

\section{Gráfico 1}

Tasa de riesgo de pobreza en la UE después de las transferencias sociales (2005-2018)

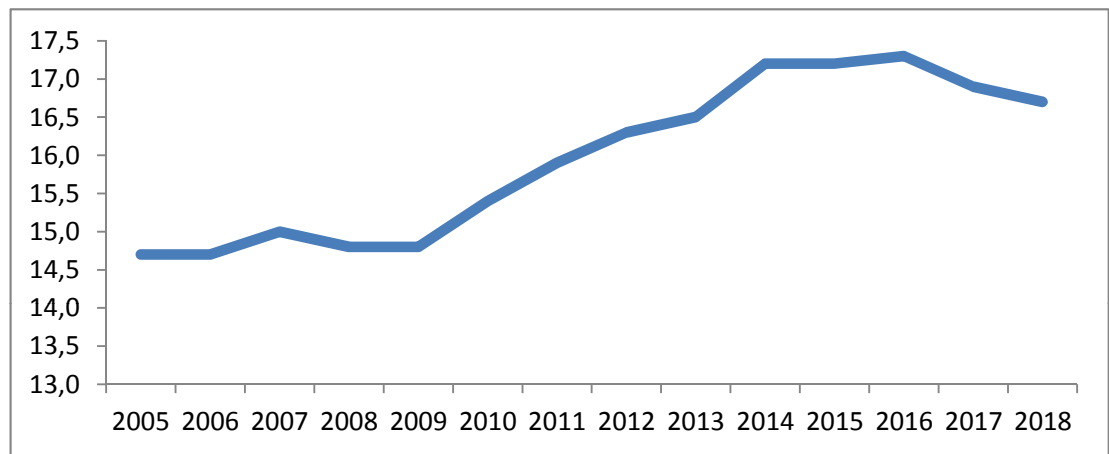

Fuente: Eurostat.

Las consecuencias del incremento de la brecha socioeconómica son diversas y casi todas ellas implican repercusiones sobre el crecimiento económico (Wilkinson y Pickett, 2019). Además, numerosas investigaciones también han señalado a la desigualdad como un importante factor criminológico debido a que aumenta la tensión social y el bloqueo de oportunidades, lo que va erosionando la cohesión social y creando controversias sociales que hacen que sea más factible y común 
la comisión de delitos ante la dificultad de establecer lazos sociales entre los miembros de la comunidad (Land, McCall y Cohen, 1990; Wilkinson, 2004; McCall, Parker y MacDonald, , 2008; McCall, 2010). Y no sólo eso, sino que otros autores, como Blau y Blau (1982), afirman que cuando aumentan las diferencias económicas entre las clases sociales de las democracias más desarrolladas se produce un aumento de las actividades delictivas y de los conflictos interpersonales que pueden ocasionar un incremento de los homicidios.

Sin embargo, a pesar de estos indicios y de los trabajos que han analizado la relación entre desigualdad y homicidios (para una revisión véase Koeppel, Rhineberger-Dunn y Mack, 2015), el estudio de esta posible vinculación ha sido relativamente escaso para la Unión Europea debido, entre otros factores, a la disponibilidad limitada de datos y a los problemas que trae consigo la comparabilidad internacional de las cifras de delincuencia (Campistol y Aebi, 2017).

De este modo, este trabajo trata de cubrir esta laguna sometiendo a contraste empírico la teoría estructural de Blau y Blau en una muestra de 25 países de la Unión Europea para el periodo 2005-2017 con el objetivo de conocer cuál es la influencia de la desigualdad originada por la crisis económica en las tasas de homicidios y determinar si el deterioro de los factores de naturaleza económica tiene incidencia o no en ella. Para ello se ha empleado un modelo de panel de datos con efectos fijos similar al de Jacobs y Richardson (2008), los cuales incluyen las variables más relevantes teóricamente.

Además, debido a las diferencias económicas, sociales y culturales existentes entre los territorios de la UE se ha decidido dividir posteriormente la muestra en cinco grupos distintos según el régimen de bienestar de los países que la componen con el fin de comprobar si los resultados obtenidos difieren según el régimen examinado. A su vez, esto permitirá analizar cómo el apoyo social brindado tanto por los gobiernos como por el sector privado a la situación económica de las familias afecta a las tasas de homicidios de los distintos países según dicha clasificación.

A continuación, en la siguiente sección, se hace un repaso de la teoría que relaciona la desigualdad y la delincuencia para, posteriormente, seguir con una revisión de la literatura académica sobre la temática investigada. En el siguiente apartado se describe la estrategia empírica 
para analizar los datos y las variables utilizadas junto con la teoría que argumenta su inclusión en el modelo econométrico empleado. La sección siguiente analiza los principales resultados tanto de la muestra general como de la división por grupos. Y, por último, se exponen las conclusiones.

\section{DESIGUALDAD ECONÓMICA Y DELINCUENCIA}

Una de las elaboraciones teóricas más conocidas desde la sociología que intenta explicar el porqué se cometen actos delictivos en las sociedades actuales es la teoría de la tensión social de Merton (1968). En ella se argumenta que cuando los estamentos sociales de una comunidad concreta no tienen la capacidad suficiente para permitir que sus miembros alcancen mediante métodos legales las metas predeterminadas culturalmente por el sistema de valores dominante, se produce un estado de anomia y de tensión social que hace que determinados sectores sociales delincan mediante el empleo de medios y acciones ilegales e ilegítimas para alcanzarlas.

En este sentido, la desigualdad actúa como un importante factor criminológico al aumentar la tensión social y el bloqueo de oportunidades, ya que, entre otras consecuencias: debilita los lazos sociales y los valores de una comunidad determinada erosionando la cohesión social (Wilkinson, 2004); reduce las posibilidades de ciertos sectores poblacionales de alcanzar las metas establecidas por la sociedad, por lo que va creciendo la presión que soportan al no poder lograr el éxito económico (LaFree, 1999); y hace que la exclusión social que sufren los sectores más desfavorecidos de la población se potencie produciendo un mayor sentimiento de desapego e indiferencia hacia las normas sociales de la comunidad a la que pertenecen (McCall y Nieuwbeerta, 2007).

Todo esto va generando sentimientos de ira, hostilidad y frustración, ya que estos estratos de la población consideran injustas e inaceptables las escasas recompensas pecuniarias del mercado laboral y las pocas oportunidades de ascender en la escala social en comparación con otros grupos más pudientes de la sociedad, lo que va formando un caldo de cultivo idóneo para producir un aumento de los actos delictivos (Blau y Blau, 1982). 
Unos de los primeros que observaron que la desigualdad influía en las tasas de homicidios y que pusieron un mayor foco de atención a los sectores más desfavorecidos en sociedades más prósperas fueron Blau y Blau (1982). Estos autores teorizan que las desigualdades relacionadas con el origen de nacimiento de las personas deterioran la integración comunitaria al crear diferencias que distancian a los distintos grupos sociales, un hecho que genera desorden social y que puede producir actos de violencia. De esta manera, parten de una visión amplia de la desigualdad, no centrándose únicamente en aspectos económicos, sino que argumentan que cuando aumentan las disparidades socioeconómicas que refuerzan las diferencias étnicas y de clases sociales de las democracias más desarrolladas, se producen conflictos personales que pueden ocasionar un incremento de los homicidios y de las posibilidades de victimización en estos grupos sociales. Esto sucede porque los sectores poblacionales afectados consideran que los procesos de obtención y distribución de riqueza son injustos e inaccesibles para ellos, lo que ocasiona dos efectos: por un lado debilita la convicción que promueven las democracias más desarrolladas de que con esfuerzo y mérito personal se puede alcanzar una buena posición social y una buena renta; mientras que, por otro lado, refuerza la idea de que son claves y determinantes otros factores externos, como puede ser haber nacido en una familia bien posicionada social y económicamente (Jacobs y Richardson, 2008).

De este modo, el crecimiento de la desigualdad que ha sufrido la Unión Europea en la última década tras la crisis económica iniciada a finales de 2007 puede hacer pensar que la tasa de homicidios se ha visto afectada por ello. Sin embargo, a pesar de que existe una amplia variedad de trabajos que han analizado esta correlación, no existe bibliografía al respecto para el caso de la Unión Europea durante este periodo. Es por ello que en la siguiente sección analizaremos la literatura académica existente para realizar una revisión del estado de la cuestión y pasar posteriormente al análisis econométrico.

\section{REVISIÓN DEL ESTADO DE LA CUESTIÓN}

La relación entre la desigualdad y los homicidios ha sido ampliamente investigada, especialmente a partir de la década de 1980 cuando comenzaron a publicarse una gran cantidad de trabajos sobre la existencia de un posible vínculo entre dichas variables. Una base importante de estas investigaciones afirmaba, tras aplicar distintos modelos econométricos 
sobre diferentes variables, que aquellos países o sociedades que tenían mayores niveles de desigualdad tendían a tener una mayor tasa de homicidios (Braithwaite y Braithwaite, 1980; Blau y Blau, 1982; Hansmann y Quigley, 1982; Avison y Loring, 1986; Messner, 1989; Gartner, Baker y Pampel., 1990; Lee, 2001).

No obstante, los trabajos que utilizan unidades muy localizadas pueden tener ciertas limitaciones debido a que en una muestra tan homogénea, la desigualdad y las diferencias de oportunidades de cada grupo social pueden ser limitadas. Además, en algunas ocasiones estas investigaciones basan sus resultados en generalidades fundamentadas en menos de 50 observaciones de casos, lo que puede producir problemas inferenciales en los análisis (Jacobs y Richardson, 2009).

Por esta razón, también destacan los estudios de carácter transnacional que deberían ser tan informativos como los anteriores, y que permiten obtener explicaciones más generales y extrapolables a otros contextos; un hecho que en el caso de las investigaciones tan localizadas puede no ocurrir al proporcionar explicaciones más específicas para una determinada realidad, por lo que no tienen por qué tener la misma capacidad explicativa en otros objetos de estudios que parten de situaciones muy distintas. En estos trabajos también se confirma en la gran mayoría de ellos que la desigualdad es un factor importante para entender los diferentes niveles de tasas de homicidios entre distintos países (LaFree, 1999; Wilkinson, 2004). Igualmente, Messner et al. (2002) o Nivette (2011) obtienen un resultado positivo en la relación desigualdad-homicidios y, además, recalcan la importancia de tener presentes variables institucionales en los modelos de desigualdad. Otro de los estudios más destacados es el de Fajnzyber et al. (2002), que investigan la relación entre la delincuencia violenta y la desigualdad para una muestra de 39 países y concluyen que ésta y las tasas de homicidios se correlacionan positivamente incluso después de controlar otras variables relacionadas con este delito.

Además, en los últimos años, la producción científica sobre esta temática se ha visto incrementada como consecuencia del crecimiento generalizado de la desigualdad en la gran mayoría de los países y la preocupación sobre los posibles efectos que esto pueda tener. Así, por ejemplo, Elgar y Aitken (2011) analizan esta problemática para un conjunto de 33 países y afirman que la asociación positiva que establece la desigualdad con los homicidios es consistente. Por otro lado, Ouimet (2012) emplea distintos factores socioe- 
conómicos en una muestra de 165 países para el 2010 y demuestra que la desigualdad, el desarrollo económico y la pobreza son buenos predictores de la tasa de homicidios.

Distintos estudios recientes también siguen estableciendo esta conexión entre estas dos variables. Santos, Testa y Weiss (2018) afirman que la desigualdad tiene una relación positiva y estadísticamente significativa con los homicidios tras analizar un grupo de 148 países. Wilkins et al. (2019), tras realizar un estudio ecológico para Canadá, Estados Unidos y Australia utilizando datos del 2011, concluyen que la desigualdad de ingresos, la inestabilidad residencial y el trabajo por cuenta propia están relacionados con este delito penal. También Mclean et al. (2019) determinan para una muestra de 142 naciones que la pobreza y la desigualdad producen mayores tasas de homicidios.

Sin embargo, también hay estudios que rechazan la existencia de una clara relación positiva entre la desigualdad y los homicidios. Entre ellos, destaca el de Neumayer (2003), que examina una muestra de 117 países durante el periodo 1980-1997 y concluye que la desigualdad no tiene una influencia positiva en los asesinatos, sino que lo que realmente incide en este delito es el crecimiento económico y la disponibilidad de encontrar empleo. Por esta razón, afirma que las relaciones positivas entre estas dos variables obtenidas en otros estudios pueden ser espurias.

Pridemore (2011) enuncia en su trabajo que la mayoría de las investigaciones que han analizado esta problemática controlan la pobreza relativa pero no la absoluta y que, sin embargo, se trata de un factor determinante en la literatura empírica para los EE.UU, razón que puede hacer pensar que los resultados obtenidos en otras investigaciones pueden verse alterados. La diferencia entre una y otra es que la privación relativa se define como el estado socioeconómico de una persona en relación con otros miembros de un mismo grupo o sociedad, mientras que la absoluta hace referencia a aquellas situaciones en las que los individuos tienen unos recursos económicos inferiores a los establecidos como mínimos para permitir disfrutar de una vida normal. Ante este hecho, Pridemore incluye esta variable en dos estudios previos, Fajnzyber, Lederman y Loayza, (2002) y Savolainen (2000), y comprueba que al tenerla en cuenta la correlación desigualdad-homicidio desaparece en dos de los tres modelos empleados. De esta manera, concluye que es necesario volver a valorar las conclusiones 
tan firmes que hay alrededor de esta asociación debido a posibles especificaciones erróneas en los modelos empleados que han podido dar lugar a resultados espurios.

Aunque en menor cantidad, también existen trabajos que han investigado esta relación para Europa en los últimos años. Así, Messner, Raffalovich y Sutton (2010), a pesar de que incluyen a Estados Unidos en su investigación, examinan esta relación para una muestra de 16 países europeos y encuentran que la privación relativa y la mortalidad infantil están vinculadas positivamente con la tasa de homicidios, pero que en cambio no hay evidencia estadística de que la pobreza absoluta incida en ella. Stickley et al. (2012) también analizan la influencia de la desigualdad socioeconómica en los homicidios de 12 países europeos y determinan que tanto la desigualdad educativa, como la absoluta y la relativa inciden positivamente sobre este tipo de delito.

Por el contrario, $\mathrm{Hu}$, Van Lenthe y MacKenbach (2015) evalúan esta correlación para una muestra de 43 países europeos en el periodo 1987-2008 y afirman que tras incluir los efectos fijos de cada país y controlar distintas variables los niveles nacionales de desigualdad de ingresos no tienen efectos sobre los homicidios. También Stamatel (2016) analiza una muestra de 33 países del viejo continente para el 2010 y concluye que, a pesar de las correlaciones bivariadas entre la desigualdad y la tasa de homicidios, tras incluir otras variables de control, esta asociación no muestra ningún resultado estadísticamente significativo.

Sin embargo, los trabajos que analizan esta correlación para la Unión Europea tras la crisis económica son prácticamente inexistentes. Así, las únicas investigaciones encontradas son las de McCall y Bauer (2014) y Stamatel (2018). La primera de ellas, a pesar de que no puede incluir a Polonia por problemas metodológicos y añade tres países que no forman parte de la UE como son Islandia, Noruega y Suiza, analiza esta relación para el resto de los países que conforman esta unión política durante el periodo que transcurre desde 1994 a 2010 y encuentra que la desigualdad económica está vinculada con las tasas de homicidios. Por su parte, Stamatel (2018) en su investigación sobre igualdad de género y tasas de victimización de homicidios femeninos para la Unión Europea, con excepción de Malta, si bien no encuentra que el índice 
de Gini tenga importancia estadística en sus análisis sí detecta que los aumentos de igualdad financiera entre hombres y mujeres reducen el riesgo de victimización violenta para ambos sexos.

De este modo, este artículo es novedoso y pretende ocupar el vacío en la literatura existente sobre los efectos de la desigualdad en la tasa de homicidios en la Unión Europea después de la crisis económica y comprobar su evolución en los últimos años.

\section{DESCRIPCIÓN DE DATOS Y METODOLOGÍA}

Para analizar la relación entre la desigualdad y la tasa de homicidios en la Unión Europea, esta investigación emplea un panel de datos con observaciones anuales para una muestra de 25 países (República Checa, Dinamarca, Alemania, Irlanda, Grecia, España, Francia, Letonia, Luxemburgo, Malta, Austria, Polonia, Portugal, Eslovenia, Eslovaquia, Finlandia, Suecia, Reino Unido, Bélgica, Estonia, Chipre, Lituania, Hungría, Holanda e Italia).

A pesar de que la UE está formada actualmente por 28 países, no se ha podido incorporar a Croacia, a Rumanía, ni a Bulgaria debido a que en sus casos no existen registros para todas las variables analizadas. Esto suele ser uno de los mayores obstáculos para ampliar las secciones transversales, lo que dificulta la realización de estudios comparativos entre países (Fajnzyber. Lederman y Loayza, 2002).

Además, siguiendo a Pampel y Gartner (1995) y a Jacobs y Richardson (2008), tan sólo se analizan los países considerados como desarrollados, ya que la inclusión de países en vías de desarrollo en el modelo, como los tres Estados nombrados anteriormente ${ }^{1}$, podría dar como resultado relaciones espurias debido a dos circunstancias: primero, por las diferencias existentes entre los determinantes del crimen para un caso y otro; $y$, segundo, debido a que existen fuertes razones tanto teóricas como metodológicas para restringir este modelo a los países más desarrollados, ya que el poder explicativo de la relación entre la desigualdad económica y los homicidios es más intenso en las sociedades más avanzadas económicamente, puesto que enfatizan las recompensas basadas en el logro de una manera más importante que en los países con economías menos desarrolladas (Pampel y Gartner, 1995; Jacobs y Richardson, 2008). 
El periodo analizado transcurre desde 2005 hasta 2017, lo que conforma un periodo prudente para comprobar si el incremento de la desigualdad y el deterioro de otras variables relacionadas con el ciclo económico durante la crisis económica y la posterior recesión influyen en la tasa de homicidios. Además, el estudio no ha podido incluir estos últimos años debido a que Eurostat tiene un retraso de dos años a la hora de proporcionar los datos sobre delincuencia, lo que imposibilita el análisis hasta el momento actual.

De este modo, este estudio ha incorporado las variables empleadas por Jacobs y Richardson (2008), que ya han sido empleadas en otras investigaciones, debido a la capacidad explicativa que pueden tener sobre la tasa de homicidios. Todas ellas han sido extraídas de Eurostat a excepción de la población urbana cuya fuente es el Banco Mundial y de la tasa de homicidios para Holanda que es proporcionada por la Oficina de las Naciones Unidas contra las Drogas y el Delito (UNODC). Las variables empleadas son las siguientes:

\section{Coeficiente de Gini}

El coeficiente de Gini se trata de la medida representativa de la desigualdad que con mayor frecuencia suele usarse en el análisis de los homicidios (Roberts y Willits, 2015). Esta variable calcula la desigualdad existente en una población a partir de los ingresos obtenidos por cada trabajador y puede oscilar entre 0 y 1 (o en escala del 0 al 100), siendo 0 la máxima igualdad posible y 1 el mayor grado de desigualdad. Se trata, por tanto, de la variable que representa más adecuadamente la situación de desigualdad en que se puede encontrar un país.

\section{Tasa de desempleo}

La tasa de desempleo se ha utilizado con frecuencia como una representación de las dificultades económicas de una determinada población con el fin de contrastar empíricamente la teoría de la tensión. Sin embargo, la mayoría de los resultados han mostrado una relación negativa con la tasa de homicidios en vez de la correlación positiva esperada, o bien no se han obtenido resultados estadísticamente significativos (McCall y Nieuwbeerta, 2007). 
La relación entre el deterioro de la economía y la delincuencia parte de la hipótesis de que el empeoramiento de los ciclos económicos, reflejado en un aumento del desempleo o en una disminución de los salarios, influirá en la motivación de los potenciales delincuentes a la hora de cometer un acto criminal (Becker, 1968). Es decir, las dificultades para acceder al mercado laboral y la disminución de las posibilidades legítimas de conseguir ingresos pueden incrementar las probabilidades de que las personas delincan con el fin de obtener dichos ingresos y así poder hacer frente a sus necesidades económicas.

Por su parte, Cantor y Land (1985) incluyeron un segundo efecto de la economía sobre la delincuencia derivado del incremento del desempleo, que explicaría los resultados negativos obtenidos en otras investigaciones sobre homicidios. Estos autores explican que los aumentos de la tasa de desempleo pueden reducir las posibles oportunidades de cometer un delito, es decir, que cabe la posibilidad de que exista una relación negativa entre estas dos variables. El razonamiento que establecen es que el descenso de la actividad laboral puede disminuir las potenciales situaciones a la hora de ser víctima de un delito. En lo que respecta a los delitos violentos, la explicación reside en que debido a que el victimario pasa más tiempo en el hogar y menos en lugares públicos, como consecuencia del empeoramiento de las condiciones económicas, está menos expuesto a potenciales situaciones de peligro (Kennedy y Forde, 1990).

\section{Mortalidad infantil}

La mortalidad infantil, como hacen otros estudios (Pare, 2006; Pridemore, 2008), se utiliza como una manera de conceptualizar la pobreza absoluta de la población debido a que se considera como un buen indicador de ella a pesar de no ser una representación exacta y directa. Sin embargo, Santos, Testa y Weiss (2018) afirman que los análisis a nivel transnacional que incluyen la privación absoluta son relativamente recientes (Cole y Gramajo, 2009; Messner, Raffalovich y Sutton, 2010; Ouimet, 2012; Rogers y Pridemore, 2013; Pare y Felson, 2014).

Esta variable es importante incluirla, ya que, como enuncia Pridemore (2011), la pobreza absoluta establece un sólido vínculo con la violencia que está respaldado por distintas teorías sociológicas y criminológicas, como la de la oportunidad, la desorganización social, la de la tensión o la teoría de las subculturas, por lo que si no es controlada 
puede dar lugar a resultados inexactos debido a una especificación errónea del modelo. En este sentido, la pobreza absoluta es un factor determinante en la literatura empírica para los EE. UU, que cuando ha sido incluida mediante medidas representativas, como la mortalidad infantil o la esperanza de vida, se han obtenido vínculos estables con los homicidios y, en cambio, ha producido inconsistencias en la relación desigualdad-homicidios.

Parte de esto se explica porque las variables que controlan la desigualdad no incluyen ciertas características de los objetos de estudios que pueden moderar los efectos de la privación relativa sobre la sociedad, como puede ser el apoyo social o las redes de protección. Además, Pridemore (2011) señala que al no ser el PIB o las variables de desigualdad medidas de la pobreza, estas no capturan la relación de este fenómeno con los homicidios. Esto ocurre porque, aunque un país pueda considerarse como rico según los indicadores económicos, una parte de su población puede estar por debajo de los umbrales de pobreza y esto puede afectar directamente los niveles de delincuencia (Ouimet, 2012).

De esta forma, las tasas de homicidios serán superiores en los países que tengan una mortalidad infantil más alta. Esto se produce porque, generalmente, habrá más familias empobrecidas, dando lugar a una mayor desorganización social y a la posibilidad de que exista por parte de la sociedad un mayor desinterés por los pobres con las consecuencias negativas que esto puede tener (Jacobs y Richardson, 2008). Además, en los países donde los niveles de pobreza sean elevados se verá reducido el grado de cumplimiento de las leyes debido a que la utilidad de cometer acciones delictivas será superior (Bourguignon, 1998).

\section{Tasa de urbanización}

La tasa de urbanización es otra de las variables empleadas en este estudio. Wirth (1938) reconoció que el tamaño y la densidad de una urbe o población incide sobre las relaciones sociales y la integración en la comunidad, a la vez que repercute tanto sobre el control social informal, ejercido por los vecinos de la comunidad, como sobre el control social formal, ejercido por las autoridades. De este modo, el debilitamiento de estos factores junto con el anonimato que otorga la 
ciudad hace más viable la comisión de actividades delictivas (Roberts y Willits, 2015), por lo que las oportunidades para cometer delitos son mayores en las urbes que en zonas rurales (Glaeser y Sacerdote, 1999).

Esta variable ha sido utilizada con bastante frecuencia en los estudios sobre homicidios en Estados Unidos, pero se han obtenido resultados mixtos y lejos de ser concluyentes, ya que algunos confirman la relación positiva entre estas dos variables (Chamlin, 1989; Sampson, 1986) y otros no presentan efectos estadísticamente significativos (Rosenfeld, Messner y Baumer, 2001; Messner, Baumer y Rosenfeld, 2004).

\section{Porcentaje de la población masculina de 15 a 29 años}

También se ha incluido el porcentaje de la población masculina de 15 a 30 años por ser la que mayoritariamente comete más delitos (Grogger, 1998; Witte, 2002).

La actividad delictiva aparece y aumenta en los primeros momentos de la adolescencia para comenzar a descender a medida que las personas envejecen debido, entre otros factores, al desarrollo de un mayor autocontrol, un descenso de la impulsividad y cambios en los estilos de vida (Loeber et al., 2008). De esta forma, una sociedad envejecida en la que la proporción de hombres jóvenes se va reduciendo debería producir un descenso en la tasa de homicidios (Jacobs y Richardson, 2008).

Sin embargo, a pesar de que la teoría relaciona esta variable con la delincuencia, existe cierta controversia respecto a los resultados obtenidos en las investigaciones sobre los homicidios, ya que distintos estudios transversales proporcionan efectos nulos (Reid et al., 2005; Maume y Lee, 2003) o incluso negativos (Land, McCall y Cohen, 1990; Lee, Maume y Ousey, 2003). Sin embargo, cuando se han empleado series temporales en los análisis, los resultados sí han sido positivos y estadísticamente significativos (Fox y Piquero, 2003).

\section{PIB per cápita}

El PIB per cápita se ha añadido como indicador del desarrollo económico. Se trata de una variable que ha sido utilizada constantemente en otros estudios y para la que se ha obtenido una sólida correlación negativa respecto a los homicidios (Fajnzyber, Lederman y Loayza, 2002; 
Lin, 2007; Altheimer, 2008). Esto es debido a que existe una relación importante entre la desigualdad y el desarrollo económico (Jacobs y Richardson, 2008), puesto que, en términos generales, los países más desarrollados deberían de tener redes de seguridad más fuertes que permitan paliar las dificultades económicas y materiales de los más desfavorecidos actuando como protectores criminológicos (Ouimet, 2012).

\section{Tasa de inmigración}

La tasa de inmigrantes legales se emplea como una representación próxima del fenómeno migratorio como hacen otros estudios (Buonanno y Montolio, 2008), y de forma análoga al empleo de las minorías que incluyen en su investigación Jacobs y Richardson (2008).

La inclusión de este factor obedece a que en los últimos años se ha investigado la relación entre el aumento de la inmigración y el de ciertos tipos de delitos (Wadsworth, 2010). La explicación que se ha dado a esta correlación es que tanto la imposibilidad de encontrar trabajo entre los inmigrantes ilegales, como los problemas de integración que pueden experimentar, aumentan las posibilidades de sufrir situaciones de precariedad y de exclusión social que pueden derivar en la comisión de delitos.

\section{Apoyo social}

El apoyo social ha sido incluido en una serie de estudios sobre los efectos de la desigualdad en los homicidios (Messner y Rosenfeld, 1997; Savolainen, 2000; Thames y McCall, 2014). El motivo de ello es que se trata de un factor que previsiblemente se espera que reduzca la tasa de delincuencia, ya que el apoyo a la situación financiera de las familias contribuye a obstaculizarla y prevenirla debido a que, entre otras consecuencias, fortalece las relaciones humanas, las redes sociales y el sentimiento de pertenencia hacia la comunidad, a la vez que mejora el control social formal e informal (Cullen, 1994).

Además, es importante destacar que el apoyo social tiene la capacidad de contener los posibles impactos negativos de la privación económica en la delincuencia. De esta forma, cuando una sociedad presenta altos 
niveles de desigualdad junto con un escaso apoyo social, se produce un efecto potenciador de la privación económica sobre el crimen (Chamlin y Cochran, 1997).

Sin embargo, en las últimas décadas las diferentes crisis económicas y los beligerantes escenarios políticos a los que han asistido los países industrializados han conducido a la privatización de numerosos servicios públicos y la reducción de sus estados de bienestar, lo que ha supuesto menores niveles de apoyo a las familias (McCall y Bauer, 2014). Esta circunstancia es importante ya que Messner y Rosenfeld (1997) sugieren que aquellos Estados que pongan mayor énfasis en la protección de sus ciudadanos durante una recesión económica tendrán tasas de homicidios más bajas. Esta hipótesis ha sido respaldada por resultados estadísticamente significativos por otras investigaciones como la de DeFronzo y Hannon (1998), la de Savolainen (2000) o la de Pratt y Godsey (2003), entre otras.

Los distintos estudios que han aplicado la teoría del apoyo social han empleado diversas medidas que por lo general limitan las posibilidades de dimensionar el concepto. Por este motivo, siguiendo a Thames y McCall (2014), se ha empleado la medida de apoyo social que proporciona Eurostat. Esta variable es definida como el gasto total anual de beneficios sociales per cápita que recoge todas las intervenciones de organizaciones públicas y privadas para aliviar a los hogares y las personas de la carga de un conjunto definido de riesgos o necesidades.

\section{ESTRATEGIA EMPÍRICA}

Esta investigación aplica un modelo similar al de Jacobs y Richardson (2008) por lo que, siguiendo a estos autores, las variables son empleadas en su forma logarítmica con la intención de corregir las posibles distorsiones que puedan causar los valores atípicos, las distribuciones asimétricas y la aparición de heterocedasticidad.

El coeficiente de Gini está en forma de promedio móvil de 3 años con el objetivo de medir su incidencia acumulativa y obtener un mayor poder explicativo que el que podrían tener los cambios a corto plazo, ya que un delito como el homicidio es muy difícil que tenga grandes oscilaciones en poco tiempo ante la modificación de una variable (Jacobs y Richardson, 2008). 
De esta forma, las variables seleccionadas han sido analizadas usando un panel de datos con efectos fijos, lo que permite incluir las características diferentes y particulares inobservables de cada país que no varían en el tiempo y controlar los posibles cambios que son estables a lo largo de los años, como pueden ser los cambios en la legislación penal. La elección de este modelo econométrico se plantea de una manera más eficaz y razonable que el uso de otros métodos, como pudiera ser un conjunto de datos de secciones transversales, debido a las características que presenta esta metodología (Hsiao y Sun, 2000).

Así, entre las ventajas de seleccionar este modelo destaca la mayor capacidad informativa de los datos de panel, ya que permiten controlar la heterogeneidad individual de los datos, tener más grados de libertad y disminuir la colinealidad entre las variables explicativas, lo que proporciona estimaciones econométricas de mayor precisión (Hsiao, 2003). Estas propiedades posibilitan controlar en el modelo las diferencias existentes entre cada país de la Unión Europea como puede ser el tamaño poblacional, la desigualdad o los ingresos, lo que puede proporcionar relaciones estadísticas más sólidas y significativas.

Además, los paneles de datos tienen mejor disposición a la hora de identificar y medir efectos que no son detectables en secciones únicamente transversales o temporales (Baltagi, 2005). La ecuación empleada es la siguiente:

$$
y_{i t}=\sum_{i=1}^{n} \delta_{i}+\beta X_{i t}+\varepsilon_{i t}
$$

Donde el índice $i$ y $t$ corresponden al país y al año. $\delta_{i}$ es el efecto fijo del país, $y_{i t}$ es la tasa de homicidios por 100.000 habitantes, $X_{i t}$ son las variables explicativas y $\varepsilon_{i t}$ es el término error. 
Gráfico 2

Tendencia en las tasas medias de homicidios en la UE (2005-2017)

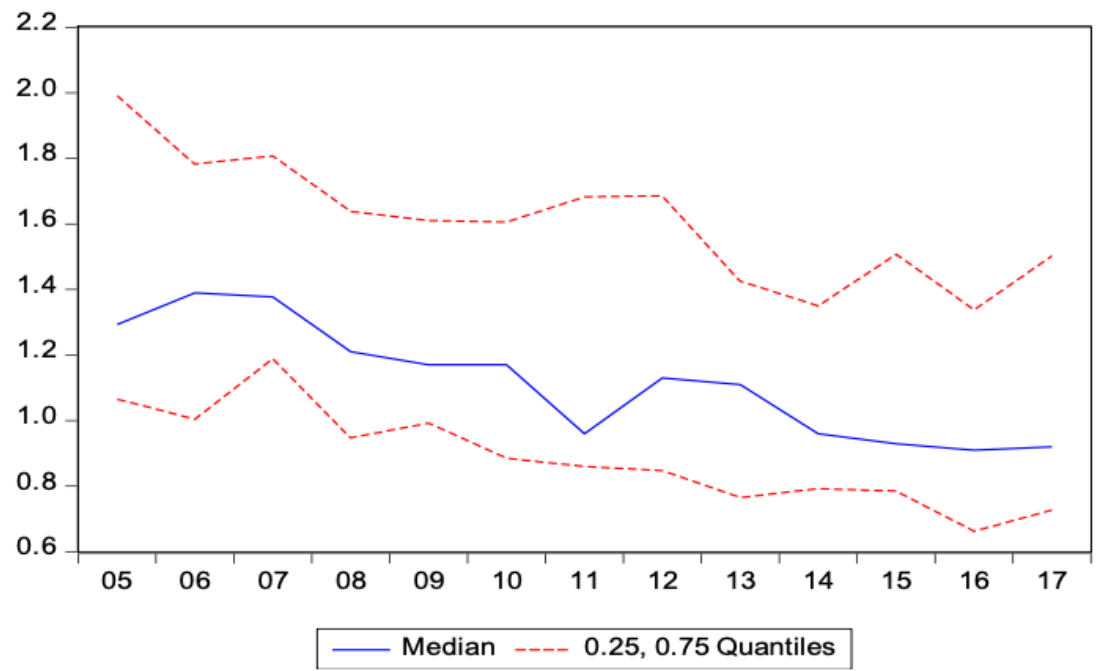

Fuente: Estimaciones del autor, a partir de la econometría y del programa Eviews.

Por último, indicar que se ha añadido el término AR(1) y AR(2) con el objetivo de eliminar la posible aparición de correlación en serie entre los residuos de la variable tasas de homicidios, ya que al tratarse de una variable cuya variación es progresiva y no tiene saltos exponenciales de un año a otro puede aparecer este problema como se puede comprobar en el Gráfico 2.

Insertando las variables empleadas en el estudio en dicha ecuación se obtiene (2):

Crimen $_{i t}=\sum_{i=1}^{n} \delta_{i}+$ Mortalidad infantil $_{i t-1}+$ Población urbana $_{i t-2}+$ Inmigración $_{i t}+$ Población masculina $_{i t}+$ PIB per capita $_{i t}+$

Desigualdad $_{i t}+$ Desempleo $_{i t-1}+\varepsilon_{i t}(2)$

\section{RESULTADOS}

El estudio econométrico está compuesto por cuatro modelos. El primero de ellos incluye todas las variables explicativas excepto la inmigración y el apoyo social; en el Modelo 2 se incluye la tasa de inmigrantes; en el Modelo 3 se utiliza la variable Gini al cuadrado con el 
fin de determinar si existe una relación no lineal entre la desigualdad y la tasa de homicidios para el periodo analizado; finalmente en el Modelo 4 se incluye la variable apoyo social.

Tabla 1

Análisis de efectos fijos de series temporales agrupadas de las tasas de asesinatos corregidas por correlación serial

\begin{tabular}{|c|c|c|c|c|c|c|c|c|}
\hline Variable & & delo 1 & Mo & delo 2 & Mos & delo 3 & Moc & lelo 4 \\
\hline PIB per cápita & $-0,461$ & {$[0,060]^{* * *}$} & $-0,405$ & {$[0,063]^{* * *}$} & $-0,397$ & {$[0,064]^{* * *}$} & $-0,298$ & {$[0,069]^{* * *}$} \\
\hline Hombres 15-29 & 0,253 & {$[0,179]^{*}$} & 0,300 & {$[0,180]^{*}$} & 0,293 & {$[0,181]^{*}$} & 0,493 & {$[0,181]^{* * *}$} \\
\hline Desempleo & $-0,095$ & {$[0,016]^{* * *}$} & $-0,095$ & {$[0,016]^{* * *}$} & $-0,095$ & {$[0,016]^{* * *}$} & $-0,104$ & {$[0,016]^{* * *}$} \\
\hline $\begin{array}{l}\text { Mortalidad } \\
\text { infantil }\end{array}$ & 0,163 & {$[0,021]^{* * *}$} & 0,162 & {$[0,021]^{* * *}$} & 0,163 & {$[0,022]^{* * *}$} & 0,163 & {$[0,021]^{* * *}$} \\
\hline Urbanización & 0,078 & {$[0,208]$} & 0,147 & {$[0,216]$} & 0,149 & {$[0,217]$} & 0,351 & {$[0,208]^{*}$} \\
\hline Inmigración & & - & $-0,089$ & {$[0,030]^{* * *}$} & $-0,091$ & {$[0,030]^{* * *}$} & $-0,043$ & {$[0,031]^{* * *}$} \\
\hline $\begin{array}{l}\text { Gini } \\
\text { (no logaritmo } \\
\text { en Modelo 3) }\end{array}$ & 0,291 & {$[0,138]^{*}$} & 0,250 & {$[0,139]^{*}$} & 0,005 & {$[0,005]$} & 0,260 & {$[0,137]^{* *}$} \\
\hline $\mathrm{Gini}^{2}$ & & - & & - & $1,80 \mathrm{E}-05$ & [6,51E-05] & & - \\
\hline Apoyo social & & - & & - & & - & $-0,242$ & {$[0,060]^{*}$} \\
\hline $\mathrm{R}^{2}$ & & 0,86 & &, 86 & &, 86 & & 85 \\
\hline AR en rezagos: & $1 \&$ & 2 años & $1 \&$ & 2 años & $1 \&$ & 2 años & $1 \&$ & años \\
\hline Durbin-Watson & & 2,04 & & 2,03 & & 03 & & 04 \\
\hline Número de obs. & & 225 & & 225 & & 225 & & 25 \\
\hline
\end{tabular}

El Modelo 1 muestra que todas las variables, excepto la población urbana, tienen efectos explicativos estadísticamente significativos en los homicidios para la muestra de los 25 países de la UE.

Como se esperaba, la desigualdad tiene una importante incidencia positiva sobre este tipo de delito y los resultados muestran que un aumento del $10 \%$ de aquella supone el incremento de casi 3 puntos porcentuales en los homicidios. Además, la pobreza absoluta, representada por la mortalidad infantil, también repercute positivamente en la variable dependiente examinada. La población masculina de 15 a 29 años igualmente se presenta como un factor del incremento de los homicidios, aunque con una significancia estadística más débil respecto a las dos variables anteriores. 
Por el contrario, al igual que se obtiene en otras investigaciones (Fajzynber, Lederman y Loayza, 2002), la mejora del PIB per cápita se sitúa como un elemento muy importante a la hora de reducir las tasas de homicidios, ya que una mejora del 10\% de esta variable llegaría a suponer casi la disminución del 5\% de los homicidios. Esto indica que el deterioro que sufrieron las economías nacionales de la gran mayoría de los países europeos durante la crisis económica y su posterior recesión incidió positivamente en el número homicidios, por lo que se extrae que una economía sana es primordial para combatir los homicidios en la Unión Europea.

Además, durante la última década las convulsiones económicas experimentadas a nivel mundial han supuesto importantes incrementos en los niveles de desempleo. Estas dificultades para acceder al mercado laboral y obtener de manera legítima los ingresos necesarios por parte de la población pueden hacer esperar un crecimiento del número de homicidios. Sin embargo, al igual que ocurre en otras investigaciones (McCall y Nieuwbeerta, 2007), los resultados obtenidos apoyan la hipótesis de Cantor y Land (1985), la cual explica que los aumentos de la tasa de desempleo pueden disminuir el número de delitos cometidos.

En el Modelo 2, tras incluir la inmigración, se siguen obteniendo los mismos resultados y valores que en el modelo anterior. En lo que respecta a la inclusión de esta nueva variable, la relación obtenida es negativa y estadísticamente significativa. Así, a pesar de que en los últimos tiempos se ha puesto el foco sobre el vínculo que pudiera existir entre inmigración y criminalidad en los países de la UE como consecuencia de la crisis de refugiados, este estudio rechaza esta hipótesis para el caso de los homicidios. Estos resultados enlazan con los de otras investigaciones para Estados Unidos en las que se concluye que la inmigración puede actuar como un efecto protector ante el crimen debido a la unión de las familias inmigrantes y al desarrollo de lazos comunitarios y de un profundo compromiso por parte de ellas con la sociedad de acogida (Wadsworth, 2010).

Los resultados obtenidos en el Modelo 3 tras incluir la variable Gini al cuadrado confirman que durante la crisis económica y los años posteriores la relación establecida entre la desigualdad y las tasas de homicidios es lineal. Esto sugiere que la relación entre estos dos factores no ha adoptado de momento la forma de $\mathrm{U}$ invertida que defienden otros autores (Jargosky, 1997; Jacobs y Richardson, 2008) y que, por tanto, al menos desde 2005 hasta 2017 para la Unión Europea estas dos variables aumentan simultáneamente a un ritmo constante. 
Finalmente, en el Modelo 4, con el objetivo de enriquecer y fortalecer el estudio se ha incluido, como hacen otras investigaciones (Thames y McCall, 2014), la variable apoyo social para comprobar si el nivel de gasto social de los países repercute sobre los homicidios. Esta variable es utilizada en forma de promedio móvil de 3 años dado que otros investigadores señalan que su influencia puede darse más bien de forma persistente que de manera inmediata (McCall y Brauer, 2014). La correlación que se obtiene es negativa, es decir, las redes de protección y el apoyo que puedan recibir las familias para mejorar su situación económica previenen los homicidios.

\section{RESULTADOS SEGÚN REGÍMENES DE BIENESTAR}

Ante estos resultados se ha decidido profundizar en el análisis con el fin de comprobar si la desigualdad y el resto de las variables afectan del mismo modo en los distintos regímenes de bienestar de la Unión Europea o si, por el contrario, las dinámicas experimentan algún tipo de variación. No obstante, es necesario indicar que este análisis tiene alguna limitación debido a la reducida secuencia de datos en algunos casos, pero, sin embargo, se trata de una primera aproximación al fenómeno que permite sacar una serie de importantes conclusiones preliminares al respecto.

Así, se parte del Modelo 4, ya que la variable apoyo social se trata de un elemento diferenciador de los sistemas de bienestar. De esta forma, el objetivo es comprobar si existen diferencias en la incidencia que este factor ejerce sobre las tasas de homicidios, puesto que dependiendo del nivel de gasto social se pueden producir distintas repercusiones sobre este delito.

En una comunidad política tan amplia, donde hay importantes diferencias económicas, sociales y culturales entre los países que la componen, es de esperar que puedan existir características particulares en término de las dinámicas delictivas.

Para la elaboración de este análisis se ha decidido dividir la muestra principal siguiendo la clasificación que estableció Esping-Andersen (1990) en modelo corporativista, liberal y socialdemócrata, y la posterior incorporación del régimen mediterráneo (Leibfried, 1992) y del transicional (Kornai, 1992). 
El modelo socialdemócrata, que engloba a los países nórdicos, aparece como una respuesta ante la política liberal y las consecuencias socioeconómicas que el libre mercado causaba en la población. Por ello, tiene como objetivos fundamentales el pleno empleo y la igualdad social, es decir, procurar que todos aquellos que conforman la sociedad tengan acceso a los recursos y medios necesarios para que puedan ser una parte activa de la comunidad y no queden excluidos de ella. De este modo, existe un acuerdo tácito entre la sociedad civil y las instituciones públicas con la finalidad de alcanzar estas metas mediante un amplio y sólido Estado de bienestar que preste los servicios necesarios, lo que supone un alto grado de desmercantilización (Esping-Andersen, 1990).

Los países pertenecientes al modelo corporativista destacan por tener un Estado bastante activo en la provisión de servicios de bienestar. No obstante, no se produce la redistribución de los ingresos desde las capas sociales más pudientes hacia los sectores de la población más necesitados, puesto que las políticas de bienestar a nivel estatal parten de la protección de la jerarquía social existente, lo que produce una notable estratificación de la sociedad. Esto ocurre porque no establecen sus fundamentos alrededor de la igualdad, sino que lo hacen en torno a la cohesión social con el fin de alcanzar dos objetivos: la seguridad y la estabilidad (Esping-Andersen, 1990). Este planteamiento se aleja de la universalidad de los servicios y de las ideas de distribución de la riqueza típicas del modelo socialdemócrata, por lo que el grado de desmercantilización es más moderado al jugar el libre mercado un papel más sustancial como proveedor de los servicios necesarios.

El modelo liberal en Europa es característico de los países anglosajones. Este régimen se caracteriza por ser el mercado el principal proveedor de los servicios y por una escasa interferencia del Estado, que generalmente se presenta como un ente vigilante encargado de garantizar que se cumplen las condiciones necesarias para el libre intercambio en el mercado, al mismo tiempo que efectúa acciones para estimularlo, ya sea de forma pasiva o activamente (Esping-Andersen, 1990). De este modo, se caracteriza por una política liberal, una economía capitalista y políticas sociales residuales, lo que conlleva a que el alcance del Estado de bienestar sea muy reducido y el nivel de transferencias bajo, dando un lugar determinante a la responsabilidad del individuo (Leibfried, 1992). Estas particularidades ponen de relieve el carácter individualista de este régimen, donde se valoran los derechos y responsabilidades 
individuales por encima de lo colectivo, y en el cual las intervenciones sociales solo afectan principalmente a los trabajadores con bajos ingresos que se encuentran en extrema necesidad (Esping-Andersen, 1990).

El modelo mediterráneo es el régimen característico de los países del sur de Europa. Esta tipología, a pesar de tener generalmente una sanidad pública universal y un sistema educativo accesible a casi todos los sectores de la población, presenta un nivel alto de mercantilización en el cual el mercado tiene un papel activo (Leibfried, 1992). La ausencia de un Estado de bienestar sólido es compensada por un fuerte familiarismo que actúa como un ancla importante en la provisión de cohesión social al encargarse de proporcionar, en la medida de lo posible, todos los servicios que las administraciones públicas no pueden proveer, lo que reduce la necesidad de acudir al mercado como única alternativa (Ferrera, 1996).

Los países del modelo transicional durante los años que formaron parte de la extinta Unión Soviética tenían un sistema económico central planificado en el cual el Estado ejercía un papel monopolista en la provisión de recursos y servicios. Sin embargo, cuando la URSS se desintegró, las reformas económicas pertinentes para que estos países pudiesen incorporarse a la órbita capitalista tuvieron como uno de sus objetivos limitar las acciones del Estado en la mayor medida de lo posible, con el fin de proteger el mercado libre sin ningún tipo de intromisión por parte de las administraciones públicas. De este modo, se caracterizan por tener un Estado de bienestar débil y poco desarrollado, con un nivel alto de mercantilización debido a que el mercado ha ocupado el puesto del Estado como proveedor principal de recursos y servicios, lo que da lugar a importantes niveles de desigualdad (Fenger, 2007).

Así, los países de la UE quedan clasificados en los distintos modelos de la siguiente manera: el corporativista, que encuadra a Alemania, Francia, Austria, Bélgica y Luxemburgo; el mediterráneo, compuesto por Grecia, España, Italia, Malta, Portugal y Chipre; el transicional, que incluye a Bulgaria, R. Checa, Letonia, Eslovenia, Eslovaquia, Estonia, Polonia y Lituania; el socialdemócrata, formado por Suecia, Finlandia y Dinamarca; y el liberal, en el que se clasifican a Irlanda y Reino Unido.

La segmentación de la muestra general en cinco subconjuntos distintos, cada uno de ellos con características propias, permitirá detectar las posibles diferencias que los contextos socioeconómicos e institucionales particulares suponen sobre los homicidios. Entendemos que 
esta escala de investigación proporciona un diagnóstico más certero de la delincuencia, vinculado a las especificidades territoriales, ya que los datos agregados invisibilizan dicha información en gran medida (Sameem y Sylwester, 2018).

Tabla 2

Análisis de efectos fijos de series temporales agrupadas de las tasas de asesinatos corregidas por correlación serial según estados de Bienestar

\begin{tabular}{|c|c|c|c|c|c|}
\hline Variable & Socialdemócrata & Corporativista & Mediterráneo & Liberal & Transicional \\
\hline $\begin{array}{l}\text { PIB per } \\
\text { cápita }\end{array}$ & $-1,116 \quad[0,864]$ & $0,930 \quad[0,697]$ & $-1,712[0.350]^{* * *}$ & $-1,763[0,366]^{* * *}$ & $1,510[0,306]^{* * *}$ \\
\hline Gini & $1,085[1,000]^{* * *}$ & $0,636 \quad[0,381]^{*}$ & $1,170[0.555]^{* *}$ & $0,930[0,817]^{* * *}$ & $0,950[0,205]^{* * *}$ \\
\hline $\begin{array}{l}\text { Hombres } \\
15-29\end{array}$ & $-1,758[0,842]^{* *}$ & $3,042[1,434]^{* *}$ & $1,881[0.424]^{* * *}$ & $-1,304[0,482]^{* * *}$ & $2,693[0,655]^{* * *}$ \\
\hline $\begin{array}{l}\text { Desem- } \\
\text { pleo }\end{array}$ & $-1,065[0,242]^{* * *}$ & $0,410[0,118]^{* * *}$ & $-0.238[0.109]^{* *}$ & $-0,593[0,098]^{* * *}$ & $0,559[0,077]^{* * *}$ \\
\hline $\begin{array}{l}\text { Mor- } \\
\text { talidad } \\
\text { infantil }\end{array}$ & $0,022 \quad[0,165]$ & $0,244[0,106]^{* *}$ & $0.158 \quad[0.098]^{*}$ & $1,357[0,661]^{* *}$ & $0,361[0,055]^{* * *}$ \\
\hline $\begin{array}{l}\text { Urbani- } \\
\text { zación }\end{array}$ & $-7,875 \quad[5,578]$ & $1,230[0,242]^{* * *}$ & $-0.090 \quad[0.231]$ & $-2,195[0,736]^{* * *}$ & $0,466 \quad[0,682]$ \\
\hline $\begin{array}{l}\text { Inmi- } \\
\text { gración }\end{array}$ & $-0,366[0,179]^{* *}$ & $0,357[0,081]^{* * *}$ & $0.083[0.118]$ & $-0,737 \quad[0,474]$ & $0,560[0,090]^{* * *}$ \\
\hline $\begin{array}{l}\text { Apoyo } \\
\text { Social } \\
\end{array}$ & $-1,055[0,543]^{* *}$ & $-3,652[1,035]^{* * *}$ & $1,121[0.175]^{* * *}$ & $-0,891 \quad[0,552]^{*}$ & $-1,999[0,256]^{* * *}$ \\
\hline $\mathrm{R}^{2}$ & 0,91 & 0,75 & 0,55 & 0,75 & 0,95 \\
\hline $\begin{array}{l}\text { AR en } \\
\text { rezagos: }\end{array}$ & $1 \& 2$ años & $1 \& 2$ años & $1 \& 2$ años & 1 año & $1 \& 2$ años \\
\hline $\begin{array}{l}\text { Durbin- } \\
\text { Watson }\end{array}$ & 2,02 & 1,91 & 2,08 & 2,2 & 2,1 \\
\hline $\begin{array}{l}\text { Número } \\
\text { de obs. }\end{array}$ & 27 & 54 & 54 & 18 & 72 \\
\hline
\end{tabular}

Fuente: Estimaciones del autor, a partir de la econometría y del programa Eviews.

Notas: SEs entre paréntesis. SEs robustos a la heterocedasticidad y autocorrelación (Arellano, 1987). *** ** $\mathrm{y}$ * indican significación al 1\%, 5\% y 10\%, respectivamente. La variable Ln Gini en esta ocasión no es calculada como promedio móvil. En el modelo Liberal todas las variables están en niveles, mientras que en el resto también están la gran mayoría en niveles menos algunas de ellas que han sido retrasadas para hacerlas estacionarias. Mediterráneo: hombres $_{t-1}$, urbanización $_{t-1}$. Transicional: hombres t-2, $_{\text {mortalidad }}$

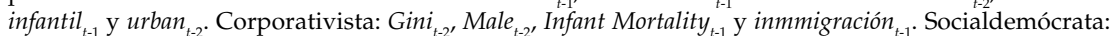
PIB per cápita $t_{t-1}$, Gini $_{t-1}$, urbanismo $_{t-1}$ y inmmigración ${ }_{t-1}$. 
Así, en la Tabla 2 se puede comprobar cómo la desigualdad está correlacionada positivamente y de manera significativa con la tasa de homicidios en todos los modelos de bienestar analizados. La pobreza, representada por la mortalidad infantil, también es un potenciador de las actividades delictivas que pueden acabar resultando letales para la vida de las personas, ya que en todos los modelos tiene una relación positiva, excepto en el socialdemócrata que no tiene significación estadística, lo que puede ser explicado por los bajos niveles de pobreza que suelen presentar estos países, que haría que esta variable no tenga incidencia en sus resultados. Estos resultados confirmarían definitivamente que para la Unión Europea la desigualdad y el aumento de la pobreza se sitúan como factores potenciales criminológicos muy importantes.

Por otro lado, el apoyo social produce los efectos negativos esperados en todos los modelos excepto en el mediterráneo, que contrariamente a la teoría establece una relación positiva con los homicidios. De este modo, se confirmaría la teoría de Cullen (1994) de que las redes de protección y el apoyo que puedan recibir las familias para mejorar su situación económica previenen los homicidios.

En lo que respecta a la incidencia positiva encontrada para el modelo mediterráneo entre el apoyo social y los delitos violentos puede deber a que en los países que componen este modelo las políticas redistributivas están orientadas normalmente hacia las clases medias y no llegan a los deciles más bajos de renta (Culfaz, 2014), por lo que su incidencia sobre la criminalidad se podría desvanecer.

La tasa de desempleo, por su parte, tiene una importancia distinta según el modelo analizado, aunque en todos, eso sí, se obtienen correlaciones significativas. De esta forma, mientras que en el sistema socialdemócrata, en el mediterráneo y en el liberal se vuelve a confirmar la hipótesis de Cantor y Land (1985), en los países del grupo transicional y en los del corporativista, por el contrario, una subida del $10 \%$ del desempleo causa un incremento en torno al $5 \%$ en los homicidios, lo que estaría acorde a los argumentos de Becker (1968).

Además, junto al desempleo el desarrollo económico también se presenta fundamental en el modelo transicional, puesto que un aumento del $10 \%$ del PIB per cápita puede incrementar hasta en 15 puntos porcentuales los niveles de homicidios. Este resultado podría encontrar su explicación en el reciente pasado comunista de estos países y el proceso de adaptación 
de sus valores culturales y de sus instituciones al sistema capitalista que ha supuesto un importante desarrollo económico para estos países, ya que como indican Jacob y Richardson (2008) el desarrollo económico tiene una relación independiente positiva con la tasa de homicidios. Por el contrario, como ocurre con la muestra total, la mejora de esta variable tiene una incidencia negativa en los homicidios de los países que son parte del sistema mediterráneo y de los que forman el liberal. Y en el caso del modelo socialdemócrata y del corporativista esta variable no presenta efectos significativos, un hecho que podría ser explicado al tratarse de los dos regímenes que más desarrollados tienen el Estado de bienestar, lo que contrarrestaría un posible deterioro de esta variable.

En lo que se refiere a la tasa de urbanización y al porcentaje de la población masculina de 15 a 29 años los resultados obtenidos son mixtos y poco concluyentes. Para los modelos mediterráneo, transicional y corporativista se obtienen los resultados esperados, ya que el aumento de este segmento poblacional produce un incremento de las tasas de homicidios. Sin embargo, para el modelo socialdemócrata y para el liberal se establece una correlación negativa y estadísticamente significativa. Esto podría dar lugar a dos hipótesis diferentes: que en los países que se incluyen en estos modelos las generaciones más jóvenes parecen ser menos violentas que sus antecesoras; o que las características típicas este tipo de delito han cambiado y ya no sólo son los hombres comprendidos entre estas edades los que más cometen esta acción.

En definitiva, los niveles de las tasas de homicidios de todos los países de la UE sin distinción alguna en función de su régimen bienestar se ven afectados de manera significativa por la desigualdad. Además, las convulsiones que ha sufrido el ciclo económico internacional en la última década también se han hecho notar en este delito, aunque si bien es cierto que dependiendo de las estructuras sociales y económicas propias de cada régimen de bienestar las oscilaciones experimentadas han sido diferentes.

\section{CONCLUSIONES}

En este estudio se han utilizado datos de 25 países de la Unión Europea para analizar los efectos de la desigualdad económica durante el periodo 2005-2017 y, a su vez, comprobar si el empeoramiento de las condiciones económicas provocado por la crisis internacional tiene influencia sobre las tasas de homicidios. 
Además, la investigación se ha enriquecido dividiendo esta muestra en cinco grupos distintos de países según sus regímenes de bienestar con el fin de comprobar si existen diferencias entre ellos tanto en la incidencia de la desigualdad como en el efecto que pueda ejercer el apoyo social $\mathrm{u}$ otras variables. Los principales resultados que se han encontrado son los siguientes.

En primer lugar, el resultado más relevante de este estudio es que la desigualdad, tras controlar una serie de variables claves según la teoría, tuvo una incidencia positiva en todos los análisis efectuados, tanto para la muestra general como para cada submuestra según el régimen de bienestar, por lo que se confirmaría el argumento teórico de Blau y Blau (1982) para las democracias más desarrolladas en el caso de la Unión Europea.

En segundo lugar, se confirma que en la Unión Europea el apoyo social tiene la capacidad de contener los posibles impactos negativos de la privación económica en la delincuencia y que se trata de un factor que coadyuva a reducir las tasas de homicidios, ya que el apoyo a la situación económica de las familias contribuye a obstaculizarla y prevenirla.

En tercer lugar, la pobreza absoluta para la Unión Europea también es un potenciador de las actividades delictivas que pueden acabar resultando letales para la vida de las personas, ya que el aumento de esta durante los años de la crisis económica establece una relación positiva y estadísticamente significativa tanto en la muestra total como en la división por regímenes de bienestar a excepción del socialdemócrata.

Por último, se confirma que existen diferencias en la incidencia de las variables analizadas sobre las tasas de homicidios según las estructuras sociales y económicas propias de cada régimen de bienestar, aunque sin lugar a duda las tasas de homicidios de todos los países de la UE se han visto afectadas de manera significativa por las convulsiones que sufrió el ciclo económico internacional en la última década. En este sentido, mientras que en la muestra general, en el sistema socialdemócrata, en el mediterráneo y en el liberal se confirma la hipótesis de Cantor y Land (1985) para la tasa de desempleo, en los países del grupo transicional y en el corporativista, por el contrario, se apoyan en los argumentos de Becker (1968). Del mismo modo, a pesar de que el desarrollo económico es determinante para reducir los homicidios en la muestra general, para los subgrupos el análisis proporciona resul- 
tados mixtos y poco concluyentes. También se han podido encontrar diferencias en la influencia de la urbanización y de la población de 15 a 29 años.

(Recibido el 15 de enero de 2020)

(Reenviado el 13 de mayo de 2020)

(Aprobado para su publicación el 2 de noviembre de 2020)

\section{NOTAS}

1. Malta (2008), Chipre (2001) y el resto de los países del este de la Unión Europea ya han dejado de ser catalogados como países en vías de desarrollo. Eslovaquia (2009), Eslovenia (2007), Estonia (2011), Hungría (estadísticas la catalogan como una nación desarrollada), Letonia (2014), Lituania (2015), Polonia (estadísticas la catalogan como una nación desarrollada) y R. Checa (2006). 


\section{REFERENCIAS}

ALTHEIMER, Irshad. (2008), "Social support, ethnic heterogeneity and homicide: A cross-national approach". Journal of Criminal Justice, vol. 36, pp. 103-114.

ARELLANO, Manuel. (1987), "Computing robust standard errors for within-groups estimators". Oxford Bulletin of Economics and Statistics, vol. 48, pp. 431-434.

AVISON, William; LORING, Pamela. (1986), "Population diversity and cross-national homicide: the effects of inequality and heterogeneity". Criminology, vol. 24, pp. 733-750.

BALTAGI, Badi H. (2005), Econometric analysis of panel data. 3rd ed., New York: John Wiley \& Sons Inc.

BECKER, Gary. (1968), "Crime and punishment: an economic approach". Journal of Political Economy, vol. 76, 169-217.

BLAU, Judith; BLAU, Peter M. (1982), "The cost of inequality: Metropolitan structure and violent crime". American Sociological Review, vol. 47, pp. 114-128.

BOURGUIGNON, François. (1998), Crime as a social cost of poverty and inequality: A review focusing on developing countries. Paris: Delta.

BRAITHWAITE, John; BRAITHWAITE, Valerie. (1980), "The effect of income inequality and social democracy on homicide". British Journal of Criminology, vol. 20, $\mathrm{n}^{\circ}$ 1, pp. 45-53.

BUONANNO, Paolo; MONTOLIO, Daniel. (2008), "Identifying the socio-economic and demographic determinants of crime across Spanish provinces". International Review of Law and Economics, vol. 28, pp. 89-97.

CAMPISTOL, Claudia; AEBI, Marcelo F. (2017), “Are juvenile criminal justice statistics comparable across countries? A study of the data available in 45 European nations". European Journal on Criminal Policy and Research, vol. 24, n 1, pp. 55-78.

CANTOR, David; LAND, Kenneth C. (1985), “Unemployment and crime rates in the post-World War II United States: a theoretical and empirical analysis". American Sociological Review, vol. 50, pp. 317-332.

CHAMLIN, Mitchell B. (1989), "A macro social analysis of the change in robbery and homicide rates: controlling the static and dynamic effects". Sociological Focus, vol. 22, pp. 275-286. ;COCHRAN, John. (1997), "Social altruism and crime". Criminology, vol. 35, pp. 203-228.

COLE, Julio; GRAMAJO, Andrés M. (2009), "Homicide rates in a cross-section of countries: evidence and interpretations". Population and Development Review, vol. 35, pp. 749-776.

CULFAZ, Eylul. (2014), "El régimen del bienestar del sur de Europa y los efectos de la crisis de 2007 en el bienestar de España”. Papeles de Europa, vol. 27, n 1, pp. 105-136.

CULLEN, Francis. (1994), "Social support as an organizing concept for criminology: presidential address to the academy of criminal justice sciences". Justice Quarterly, vol. 11, pp. 527-559.

DEFRONZO, James; HANNON, Lance. (1998), “Welfare assistance levels and homicide rates. Homicide Studies, vol. 2, pp. 31-45. 
ELGAR, Frank; AITKEN, Nicole. (2011), "Income inequality, trsut and homicide in 33 countries". European Journal of Public Health, vol. 21, n 2, pp. 241-246.

ESPING-ANDERSEN, Gosta. (1990), The three worlds of wefare capitalism. Cambridge: Polity press.

EUROPEAN CENTRAL BANK. (2016), "Household finance and consumption network". ECB Statistics Paper, $\mathrm{n}^{\circ} 18$.

FAJNZYBER, Pablo; LEDERMAN, Daniel; LOAYZA, Norman. (2002), "Inequality and violent crime". Journal of Law and Economics, vol. 45, $\mathrm{n}^{\circ} 1$, pp. 1-40.

FENGER, Menno. (2007), "Welfare regimes in central and Eastern Europe: Incorporating post-communist countries in a welfare regime typology". Contemporary Issues and Ideas in Social Sciences, vol. 3, n 2, pp. 1-30.

FERRERA, M. (1996), "The 'Southern' model of Welfare in Social Europe". Journal of European Social Policy, vol. 6, nº 1, pp. 17-37.

FOX, James; PIQUERO, Alex R. (2003), “Deadly demographics: population characteristics and forecasting homicide trends". Crime \& Delinquency, vol. 49, pp. 339-359.

GARTNER, Rosemary; BAKER, Kathryn; PAMPEL, Fred C. (1990), "Gender stratification and the gender gap in homicide victimization". Social Problems, vol. 37, pp. 593-612.

GLAESER, Edward; SACERDOTE, Bruce. (1999), "Why is there more crime in cities?" Journal of Political Economy, vol. 107, pp. 225-258.

GROGGER, Jeff. (1998), "Market wages and youth crime". Journal Labor Economics, vol. 16, $\mathrm{n}^{\circ} 44$, pp. $756-791$.

HABERMAS, Jürgen. (2012), The crisis of European Union: a response. Malden: Polity Press.

HANSMANN, Henry; QUIGLEY, John M. (1982), "Population heterogeneity and the sociogenesis of homicide". Social Forces, vol. 61, pp. 206-224.

HSIAO, Cheng. (2003), Analysis of Panel Data. Cambridge: Cambridge University Press. ; SUN, Baohong. (2000), “To pool or not to pool panel data, panel data econometrics: future directions". Papers in Honor of Professor Pietro Balestra, edited by J. Krishnakumar and E. Ronchetti. Amsterdam: North Holland.

HU, Yannan; VAN LENTHE, Frank; MACKENBACH, Johan P. (2015), "Income inequality, life expectancy and cause-specific mortality in 43 European countries, 1987-2008: a fixed effects study". European Journal Epidemiology, vol. 20, nº 8, pp. 615-625.

JACOBS, David; RICHARDSON, Amber M. (2009), "Economic inequality and homicide in the developed nations from 1975 to 1995". Homicide Studies, vol. 12, n 1, pp. 28-45.

JARGOSKY, Paul A. (1997), "Take the money and run: economic segregation in U.S. metropolitan areas". American Sociological Review, vol. 61, pp. 984-998.

KENNEDY, Leslie; FORDE, David R. (1990), "Routine activities and crime: an analysis of victimization in Canada". Criminology, vol. 28, pp. 137-152.

KOEPPEL, Maria; RHINEBERGER-DUNN, Gayle; MACK, Kristin Y. (2015), “Cross-national homicide: a review of the current literature". International Journal of Comparative and Applied Criminal Justice, vol. 39, $\mathrm{n}^{\circ}$ 1, pp. 47-85.

DADOS, Rio de Janeiro, vol.65 (1): e20200011, 2022 
KORNAI, Janos. (1992), The socialist system: the political economy of communism. Oxford: Oxford University Press.

LAFREE, Gary. (1999), "A summary and review of cross-national comparative studies of homicide”. In: M. Smith; M. Zahn (eds.), Homicide: a sourcebook of social research. Thousand Oaks, CA: Sage.

LAND, Kenneth; MCCALL, Patricia; COHEN, Lawrence E. (1990), "Structural covariates of homicide rates: Are there any invariances across time and social space?" American Journal of Sociology, vol. 96, pp. 923-963.

LEE, Matthew. (2001), "Population growth, economic inequality, and homicide". Deviant Behavior: An Interdisciplinary Journal, vol. 22, pp. 491-516.

; MAUME, Michael; OUSEY, Graham C. (2003), "Social isolation and lethal violence across the metro/nonmetro divide: the effects of socioeconomic disadvantage and poverty concentration on homicide". Rural Sociology, vol. 68, pp. 107-131.

LEIBFRIED, Stephan. (1992), “Towards a European Welfare State?: on integrating poverty regimes into the European Community". In: Z. Ferge; J. E. Kolberg (eds.), Social policy in a changing Europe. Frankfurt: Westview Press.

LIN, Ming-Jen. (2007), "Does democracy increase crime? The evidence from international data". Journal of Comparative Economics, vol. 35, pp. 467-483.

LOEBER, Rolf et al. (2008), Violence and serious theft: development and prediction from childhood to adulthood. New York: Routledge.

MAUME, Michael; LEE, Matthew. (2003), “Social institutions and violence: A subnational test of institutional anomie theory". Criminology, vol. 4, pp. 1137-1172.

MCCALL, Patricia. (2010), "The Invariance of structural covariates: reflections on 20 years of homicide research". Homicide Studies, vol. 14, n 3, pp. 215-218.

MCCALL, Patricia; BRAUER, Jonathan R. (2014), "Social welfare support and homicide: longitudinal analyses of European countries from 1994 to 2010". Social Science Research, vol. 48 , pp. $90-107$.

MCCALL, Patricia; NIEUWBEERTA, Paul. (2007), "Structural covariates of homicide rates. A European City Cross-National Comparative Analysis". Homicides Studies, vol. 11, n ${ }^{\circ}$ 2, pp. 167-188.

; PARKER, Karen F.; MACDONALD, John M. (2008), “The dynamic relationship between homicide rates and social, economic, and political factors from 1970 to 2000 ". Social Science Research, vol. 37, n 3, pp. 721-735.

MCLEAN, Craig et al. (2019), "Exploring the relationship between neoliberalism and homicide: a cross-national perspective". International Journal of Sociology, vol. 49, nº 1, pp. 53-76.

MERTON, Robert K. (1968), Social theory and social structure. New York: Free Press.

MESSNER, Steven. (1989), "Economic discrimination and societal homicide rates: Further evidence on the cost of inequality". American Sociological Review, vol. 54, pp. 597-611.

; BAUMER, Eric; ROSENFELD, Richard. (2004), “Dimensions of social capital and rates of criminal homicide". American Sociological Review, vol. 69, pp. 882-903. 
MESSNER, Steven; RAFFALOVICH, Lawrence; SHROCK, Peter. (2002), "Reassessing the cross-national relationship between inequality and homicide rates: implications of data quality control in the measurement of income distribution". Journal of Quantitative Criminology, vol. 18, pp. 377-395.

MESSNER, Steven; RAFFALOVICH, Lawrence; SUTTON, Gretchen. (2010), “Poverty, infant mortality, and homicide rates in cross-national perspective: Assessments of criterion and construct validity". Criminology, vol. 48, pp. 509-537.

MESSNER, Steven; ROSENFELD, Richard. (1997), "Political restraint of the market and levels of criminal homicide: a cross-national application of institutional-anomie theory". Social Forces, vol. 75, pp. 1393-1416.

NEUMAYER, Eric. (2003), “Good policy can lower violent crime: evidence from a cross-national panel of homicide rates, 1980-1997". Journal of Peace Research, vol. 40, n 6, pp. 619-640.

OECD (Organisation for Economic Co-operation and Development). (2017), Understanding the socio-economic divide in Europe. Background Report.

OUIMET, Marc. (2012), "A world of homicides: the effect of economic development, income inequality, and excess infant mortality on the homicide rate for 165 countries in 2010". Homicide Studies, vol. 16, nº 3, pp. 238-258.

PAMPEL, Fred; GARTNER, Rosemary. (1995), “Age structure, socio-political institutions, and national homicide rates". European Sociological Review, vol. 11, pp. 243-260.

PARE, Paul. (2006), Income inequality and crime across nations reexamined. Doctoral dissertation, Pennsylvania State University, University Park.

; FELSON, Richard. (2014), "Income inequality, poverty and crime across nations". The British Journal of Sociology, vol. 65, pp. 434-458.

PIRRO, Andrea; TAGGART, Paul; VAN KESSEL, Stijn. (2018), “The populist politics of Euroscepticism in times of crisis: Comparative conclusions". Politics, vol. 38, n 3, pp. 378-390.

PRATT, Travis; GODSEY, Timothy. (2003), "Social support, inequality and homicide: a cross-national test of an integrated theoretical model". Criminology, vol. 41, nº 3, pp. 611-643.

PRIDEMORE, William. (2008), “A methodological addition to the cross-national empirical literature on social structure and homicide: a first test of the poverty-homicide thesis". Criminology, vol. 46, nº 1, pp. 133-154.

. (2011), "Poverty matters: a reassessment of the inequality-homicide relationship in cross-national studies". British Journal of Criminology, vol. 51, pp. 739-772.

REID, Lesley et al. (2005), “The immigration-crime relationship: evidence across US metropolitan áreas". Social Science Research, vol. 34, pp. 757-780.

SANTOS, Mateus; TESTA, Alexander; WEISS, Douglas. (2018), "Where poverty matters: examining the cross-national relationship between economic deprivation and homicide". The British Journal of Criminology, vol. 58, n² 2, pp. 372-393.

ROBERTS, Aki; WILLITS, Dale. (2015), "Income inequality and homicide in the United States: consistency across different income inequality measures and disaggregated homicide types". Homicide Studies, vol. 19, no 1, pp. 28-57. 
ROGERS, Meghan; PRIDEMORE, William. (2013), "The effect of poverty and social protection on national homicide rates: direct and moderating effects". Social Science Research, vol. 42 , pp. 584-595.

ROSENFELD, Richard; MESSNER, Steven; BAUMER, Eric P. (2001), "Social capital and homicide". Social Forces, vol. 80, pp. 283-309.

SAMEEM, Sediq; SYLWESTER, Kevin. (2018), “Crime during the business cycle: urban-rural differences". Applied Economics, vol. 50, n² 22, pp. 2500-2508.

SAMPSON, Robert J. (1986), "Neighborhood family structure and the risk of personal victimization". In: J. M. Byrne; R. J. Sampson (eds.), The social ecology of crime. New York: Springer-Verlag.

SAVOLAINEN, Jukka. (2000), “Inequality, welfare state and homicide: Further support for the institutional anomie theory". Criminology, vol. 38, n 4, pp. 1021-1042.

STAMATEL, Janet P. (2016), “Democratic cultural values as predictors of cross-national homicide variation in Europe". Homicide Studies, vol. 20, pp. 239-256.

. (2018), "Money matters: dissecting the relationship between gender equality and female homicide victimization rates in the European Union". Feminist Criminology, vol. 13, n 5 , pp. 435-455.

STICKLEY, Andrew et al. (2012), "Socioeconomic inequalities in homicide mortality: a population-based comparative study of 12 European countries". European Journal of Epidemiology, vol. 27, $\mathrm{n}^{\circ} 11$, pp. 877-884.

THAMES, Kelly; MCCALL, Patricia. (2014), "A longitudinal examination of the effects of social support on homicide across European Regions". International Journal of Conflict and Violence, vol. 8, $\mathrm{n}^{\circ}$ 2, pp. 243-261.

WADSWORTH, Tim. (2010), "Is immigration responsible for the crime drop? An assessment of the influence of immigration on changes in violent crime between 1990 and 2000". Social Science Quarterly, vol. 91, n 2, pp. 531-553.

WILKINS, Natalie et al. (2019), "Societal determinants of violent death: the extent to which social, economic, and structural characteristics explain differences in violence across Australia, Canada, and the United States". SMM-Populaton Health, vol. 8, pp. 1-8.

WILKINSON, Richard. (2004), "Why is violence more common where inequality is greater?" Annals of the New York Academy of Sciences, vol. 1036, n 1, pp. 1-12.

; PICKETT, Kate. (2019), The inner level: how more equal societies reduces stress, restore sanity and improve. Madrid: Capitan Swing.

WIRTH, Louis. (1938), “Urbanism as a way of life”. American Journal of Sociology, vol. 44, pp. 1-24.

WITTE, Ann. (2002), Crime causation: economic theories, in Encyclopedia of Crime and Justice. New York: Macmillan Reference USA. 


\section{RESUMO \\ O Efeito da Desigualdade Econômica nos Homicídios na União Europeia}

Este artigo pesquisa, a partir da teoria de Blau e Blau (1982), a relação entre a desigualdade e os homicídios na União Europeia após a última crise econômica de 2008. Para isso, utiliza-se um painel de dados com efeitos fixos no qual é utilizada a média móvel da desigualdade para capturar seus efeitos acumulativos. Os resultados indicam que a desigualdade tem uma incidência positiva em todas as análises feitas, confirmando os argumentos de Blau e Blau. Além disso, a pobreza absoluta também é um importante potenciador da taxa de homicídios na UE. Igualmente, a amostra é dividida em cinco grupos segundo os regimes de bem-estar de cada país para controlar as diferenças entre países e se detectam algumas divergências na incidência das variáveis analisadas sobre os homicídios. Finalmente, determina-se que o apoio social é um fator que contribui para a redução desse delito.

Palavras-chave: desigualdade econômica; taxa de homicídios; União Europeia; crise econômica; regimes de bem-estar

\section{ABSTRACT \\ The Effect of Economic Inequality on Homicides in the European Union}

Based on the work of Blau and Blau (1982), this article studies the correlation between inequality and homicides in the European Union after the last economic crisis of 2008. In order to attain this goal, we use a data panel with fixed effects in which the inequality moving average is used to capture its cumulative effects. The results show that inequality has a positive effect on every analysis made, confirming Blau and Blau's reasoning. Furthermore, absolute poverty is also an important homicide rate enhancer in the EU. Moreover, the sample is divided into five groups under the welfare regime of every country to control the differences, and some divergences on the effect of the variables analyzed about homicides have been detected. Finally, it is determined that social support is a factor that contributes to minimizing this type of crime.

Keywords: economic inequality; murder rates; European Union; economic crisis; welfare systems 


\section{RÉSUMÉ \\ L'Effet des Inégalités Économiques sur les Homicides dans l'Union Européenne}

Basé sur la théorie de Blau et Blau (1982), cet article étudie la relation entre les inégalités et les homicides dans l'Union Européenne après la dernière crise économique de 2008. Pour ce faire, on utilise un panel de données à effets fixes, dans lequel la moyenne mobile des inégalités est utilisée pour capturer ses effets cumulés. Les résultats indiquent que l'inégalité a un impact positif sur toutes les analyses réalisées, confirmant les arguments de Blau et Blau. En outre, la pauvreté absolue est également un facteur important d'augmentation du taux d'homicides dans l'UE. Également, l'échantillon est divisé en cinq groupes, selon les régimes de protection sociale de chaque pays pour contrôler les différences entre pays et détecter certaines divergences dans l'incidence des variables analysées sur les homicides. Enfin, il est déterminé que le soutien social est un facteur qui contribue à réduire cette criminalité.

Mots-Clés: inégalité économique; taux de meurtres; Union Européenne; crise économique; systèmes de protection sociale

\section{RESUMEN}

\section{El Efecto de la Desigualdad Económica en los Homicidios en la Unión Europea}

Este artículo investiga a partir de la teoría de Blau y Blau (1982) la relación entre la desigualdad y los homicidios en la Unión Europea tras la última crisis económica de 2008. Para ello se emplea un panel de datos con efectos fijos en el que se utiliza el promedio móvil de la desigualdad para capturar sus efectos acumulativos. Los resultados indican que la desigualdad tiene una incidencia positiva en todos los análisis hechos, confirmándose las argumentaciones de Blau y Blau. Además, la pobreza absoluta también es un importante potenciador de la tasa de homicidios en la UE. Asimismo, la muestra se divide en cinco grupos según los regímenes de bienestar de cada país para controlar las diferencias entre países y se detectan ciertas divergencias en la incidencia de las variables analizadas sobre los homicidios. Finalmente, se determina que el apoyo social es un factor que coadyuva a reducir este delito.

Palabras clave: desigualdad económica; tasa de homicidios, Unión Europea, crisis económica, regímenes de bienestar 